\title{
Search for Supersymmetry with R-Parity violation at LEP
}

M.C. Espirito Santo* CERN, Geneva, Switzerland

E-mail: 'maria.espirito.santo@cern. ch'

ABstRACT: Supersymmetry with R-parity violation has been searched for at LEP in a large number of channels and final state topologies, at centre-of-mass energies up to $209 \mathrm{GeV}$. In this presentation these searches are motivated and their results reported. No signal was found in any of the channels and limits at 95\% CL on cross-sections and masses were derived. All results are preliminary

\section{Introduction}

Supersymmetry (SUSY) is the theory behind most particle searches at colliders. R-parity is a discrete, multiplicative symmetry related to matter parity:

$$
R_{p}=(-1)^{2 S+3 B+L}
$$

where $S$ is the spin of the particle and $B$ and $L$ are the barion and lepton number, respectively. In the SUSY searches at LEP R-parity conservation $(\mathrm{RpC})$ is most frequently assumed. Scenarios with R-parity violation (RpV) will be considered in this presentation. In fact, $\mathrm{RpC}$ is not required neither by SUSY nor by Gauge Invariance. On the other hand, it is interesting to know how dependent the complex SUSY exclusions derived at LEP are on the $\mathrm{RpC}$ assumption. Finally, RpV could produce some striking signatures (many jets and/or leptons, large visible energy) and be a good way to discover SUSY.

\section{Explicit R-parity violation}

R-parity can be explicitly violated [i] $\left[\begin{array}{l}1 \\ ]\end{array}\right.$ by introducing the following trilinear terms in the superpotential:

$$
\lambda_{i j k} L_{L}^{i} L_{L}^{j} \bar{E}_{R}^{k}+\lambda_{i j k}^{\prime} L_{L}^{i} Q_{L}^{j} \bar{D}_{R}^{k}+\lambda_{i j k}^{\prime \prime} \bar{U}_{R}^{i} \bar{D}_{R}^{j} \bar{D}_{R}^{k},
$$

\footnotetext{
${ }^{*}$ Speaker.
} 
where $L_{L}, Q_{L}\left(E_{R}, U_{R}, D_{R}\right)$ are left-handed (right-handed) lepton and quark doublets (singlets) and $i, j, k$ are family indices. This leads to 45 new couplings, violating $\mathrm{L}$ ( $\lambda$ and $\left.\lambda^{\prime}\right)$ or B $\left(\lambda^{\prime \prime}\right)$ conservation.

The occurrence of $\mathrm{RpV}$ has some major phenomenological consequences. SUSY particles (sparticles) can be singly produced, and can decay directly into SM particles. The Lightest SUSY Particle (LSP) is no longer stable, and can be charged or neutral. This different phenomenology, with many new possible topologies and not necessarily preserving the traditional missing energy signature, makes that the limits derived under the $\mathrm{RpC}$ assumption cannot be taken for granted.

Although $\mathrm{RpV}$ is still an open possibility, limits on its coupling parameters exist already, from the non-observation of a certain number of effects in Standard Model (SM) processes (e.g. lepton universality violation, fast proton decay). The order of magnitude of these limits varies with the $\lambda$ coupling considered (in most of the cases they are roughly in the range $\left.1-10^{-2}\right)$. Very stringent limits exist on the simultaneous presence of certain couplings $\left(\lambda_{11 k}^{\prime} \times \lambda_{11 k}^{\prime \prime}<10^{-24}\right)$.

At LEP, SUSY with RpV is searched for both in sparticle production and decay. In the first case single sneutrino production through either $e^{+} e^{-} \rightarrow \tilde{\nu}[2]$ or $e^{+} \gamma \rightarrow \tilde{\nu} \ell\left[\hat{3}_{i}^{i}\right]$ are considered. In the second case, $\mathrm{RpC}$ sfermion and gaugino pair production is considered

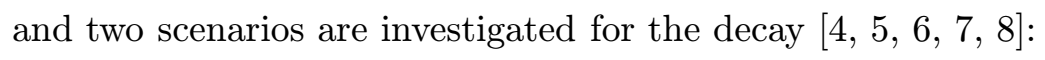

- direct decays: $\mathrm{RpV}$ decays of sparticles into SM fermions, e.g. $\tilde{f} \rightarrow f f$ and $\chi_{1}^{0} \rightarrow$ $\tilde{f}^{*} f \rightarrow f f f$

- indirect decays: cascade $(\mathrm{RpC})$ decays of sparticles into the LSP, which decays via $\mathrm{RpV}$, e.g. $\chi_{1}^{ \pm} \rightarrow W \chi_{1}^{0} \rightarrow f \tilde{f}^{*} \rightarrow f f f$.

It is assumed in all cases that only one of the $\lambda$ couplings is non-negligible at a time and that sparticles decay promptly $(\sim 1 \mathrm{~cm})$. This limits the sensitivity on $\lambda$ to values of the order of $10^{-5}$. In these searches the minimal particle content of the Minimal Supersymmetric Standard Model (MSSM) with gravity mediated SUSY breaking is assumed. Further assumptions (in particular gaugino mass unification at the GUT scale) are relevant for some of the interpretations.

These searches are characterized by the fact that a very large number of channels and final state topologies have to be covered and combined. The challenges in the analyses range from the treatment of topologies with many jets to lepton and in particular tau identification (crucial to identify some striking signatures). Usually several topological analyses are developed and combined. Results are given for the different couplings or choosing the worst case. While the background from SM processes is very dependent on the channel, 4 -fermions final states are in many cases the most relevant ones. Signal simulation was produced for different relevant combinations of parameters using SUSYGEN2.2 (3.0) [i.j].

All the data collected in the second phase of LEP were analysed, in a total of about 714 $\mathrm{pb}^{-1}$ per experiment at centre-of-mass energies ranging from 130 to $209 \mathrm{GeV}$. No evidence for a signal was observed in any of the channels and 95\% confidence level (CL) limits were 
derived. All results are preliminary. An exhaustive presentation of the results can be found in the references [2] to [8]

Considering the sfermion pair production with direct $\mathrm{RpV}$ decay, the final state topologies arising for the different types of sfermions and couplings range from 2 acoplanar leptons to 4 jets. In particular, the 4 -jets final state plays a role in the search for all sfermion types, since it is the expected final state for different types of couplings $\left(\lambda^{\prime}\right.$ in the case of sleptons and sneutrinos, $\lambda^{\prime \prime}$ in the case of squarks). Similar analyses can be used in the different cases. Figure $\prod_{1}^{\bar{i}}$ shows the cross-section limits obtained by ALEPH, together with expected MSSM cross-sections. Mass limits for the different sfermion types roughly in the range $75-85 \mathrm{GeV} / c^{2}$ can be derived from the figure.

If indirect sfermion decay modes are considered, the selection efficiencies are expected to depend on the difference between the sfermion mass and the mass of the LSP. Crosssection limits for stau pair production obtained by L3 in the plane of the two masses are shown in figure $2 i_{-i}^{-}$Contrary to what happens under the assumption of $\mathrm{RpC}$, if $\mathrm{RpV}$ occurs sneutrinos are expected to decay visibly in most of the parameter space. Figure $\underline{3}_{1}$, shows the excluded region in the plane of the sneutrino - lightest neutralino masses obtained by DELPHI assuming sneutrino pair production and indirect RpV decay through a $\lambda$ type coupling. Similar results for sleptons and squarks, and for other types of couplings, can be found in the references. Single sneutrino production through $\mathrm{RpV}$ were also searched for. The results can be found in [20, explored mass range, the results are limits on the coupling parameter, which in this case appears at the production vertex.

Considering now the pair production of charginos and neutralinos, in the MSSM parameter space regions not excluded by LEP1 data, indirect decay modes are expected to dominate, with $\chi^{ \pm} \chi^{ \pm} \rightarrow W W \chi^{0} \chi^{0}$ and the neutralinos then decaying through $\mathrm{RpV}$ into final states with 4 leptons and missing energy, 4 jets and leptons or 6 jets, depending on the coupling. This leads to complex topologies with up to 10 jets in the final state. The cross-section limits obtained in these channels can be translated into exclusions in the MSSM parameter space. Figure ${ }_{-1}^{-1}$ shows the exclusion obtained by ALEPH in the $\left(\mathrm{M}_{2}, \mu\right)$ plane, for $\tan \beta=1.4$ and a high value of $\mathrm{m}_{0}$, assuming the presence of a $\lambda^{\prime} \mathrm{RpV}$ coupling. Exclusions of this type can be converted into limits on the masses of sparticles. The mass limits obtained by L3 for $\chi_{1}^{0}, \chi_{2}^{0}$ and $\tilde{\ell}$, as a function of $\tan \beta$, considering a low value of $\mathrm{m}_{0}$ and RpV couplings of type $\lambda$ and $\lambda^{\prime \prime}$, are shown in figure RpV SUSY searches allow to exclude chargino masses up to $103 \mathrm{GeV} / c^{2}$ and LSP masses up to $38-40 \mathrm{GeV} / c^{2}$.

\section{Spontaneous R-parity violation}

R-parity could be spontaneously broken through a right-handed sneutrino acquiring a nonzero vacuum expectation value [1] term, $\epsilon_{i} L_{I} H_{u}$. In such scenarios a massless majoron $\mathrm{J}$ may arise and, through leptongaugino mixing, the decay $\chi_{1}^{ \pm} \rightarrow \tau^{ \pm} J$ can be important (specially for large values of the violation parameter $\epsilon$ ). Under the assumption of mixing in the third family, chargino pair 
production and decay into a majoron and a tau lepton, with a signature of 2 acoplanar taus were searched for.

The data collected at centre-of-mass energies up to $202 \mathrm{GeV}$ were analysed by DELPHI and no signal was observed in this channel. A cross-section limit of $0.14 \mathrm{pb}$ was obtained, corresponding to a limit on the chargino mass of $100.9 \mathrm{GeV} / c^{2}$, as shown in figure ' $\mathbf{6}_{-}^{\mathrm{i}}$. Exclusions on the MSSM parameter space were also derived in this scenario.

\section{Conclusions}

SUSY with RpV was searched for at LEP in a large number of channels and final state topologies. No signal was found, and 95\% CL limits were derived on cross-sections, masses and $\mathrm{RpV}$ couplings. In general the excluded ranges are comparable for those obtained under the assumption of RpC. For comparable scenarios, chargino exclusion up to near the kinematic limit $\left(101 \mathrm{GeV} / c^{2}\right.$ in the case of spontaneous $\left.\mathrm{RpV}\right)$ and an LSP mass limit of the order of $40 \mathrm{GeV} / c^{2}$ were obtained.

\section{Acknowledgments}

I would like to thank warmly all the people contributing to the excellent performance of the LEP collider and the four LEP collaboration for making available their latest results.

\section{References}

[1] P.Fayet, Phys. Lett. B69 (1977) 489;

G.Farrar and P.Fayet, Phys. Lett. B76 (1978) 575.

[2] DELPHI Collab., "Search for resonant sneutrino production at $\sqrt{s}=202-208 \mathrm{GeV}$ ", DELPHI 2001-019 CONF 460, March 2001.

[3] ALEPH Collab., in Proceedings of the EPS International Conference on High Energy Physics, Budapest, 2001 (D. Horvath, P. Levai, A. Patkos, eds.), JHEP (http://jhep.sissa.it/) Proceedings Section, PrHEP-hep2001/228.

[4] ALEPH Collab., in Proceedings of the EPS International Conference on High Energy Physics, Budapest, 2001 (D. Horvath, P. Levai, A. Patkos, eds.), JHEP (http://jhep.sissa.it/) Proceedings Section, PrHEP-hep2001/230.

[5] ALEPH Collab., in Proceedings of the EPS International Conference on High Energy Physics, Budapest, 2001 (D. Horvath, P. Levai, A. Patkos, eds.), JHEP (http://jhep.sissa.it/) Proceedings Section, PrHEP-hep2001/226.

[6] DELPHI Collab., in Proceedings of the EPS International Conference on High Energy Physics, Budapest, 2001 (D. Horvath, P. Levai, A. Patkos, eds.), JHEP (http://jhep.sissa.it/) Proceedings Section, PrHEP-hep2001/331.

[7] L3 Collab., in Proceedings of the EPS International Conference on High Energy Physics, Budapest, 2001 (D. Horvath, P. Levai, A. Patkos, eds.), JHEP (http://jhep.sissa.it/) Proceedings Section, PrHEP-hep2001/540. 
[8] OPAL Collab., in Proceedings of the EPS International Conference on High Energy Physics, Budapest, 2001 (D. Horvath, P. Levai, A. Patkos, eds.), JHEP (http://jhep.sissa.it/) Proceedings Section, PrHEP-hep2001/27.

[9] S.Katsanevas and P.Morawitz, Comp.Phys Comm., 112 (1998) 227.

[10] J.C.Romao et al., Phys. Lett. B288 (1992) 311.

[11] DELPHI Collab., in Proceedings of the EPS International Conference on High Energy Physics, Budapest, 2001 (D. Horvath, P. Levai, A. Patkos, eds.), JHEP (http://jhep.sissa.it/) Proceedings Section, PrHEP-hep2001/332. 


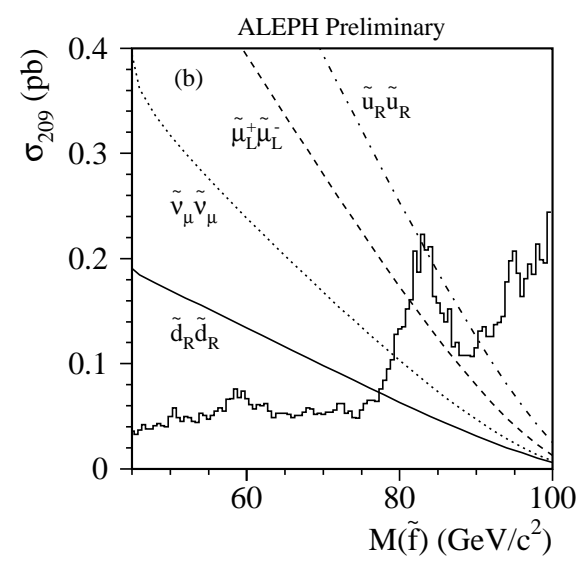

Figure 1: Excluded cross-sections for sleptons (via a $\lambda^{\prime}$ type coupling), sneutrinos (via $\lambda^{\prime}$ ) and squarks (via $\lambda^{\prime \prime}$ ) decaying directly to 4 jets (histogram). The MSSM cross-sections are superimposed (lines).

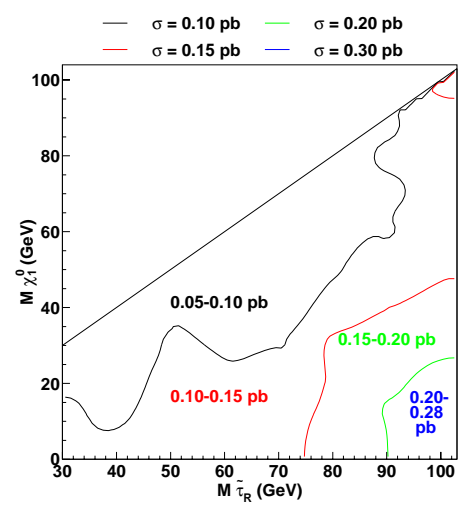

Figure 2: Stau pair production cross-section limit by the L3 collaboration for indirect RpV decay.

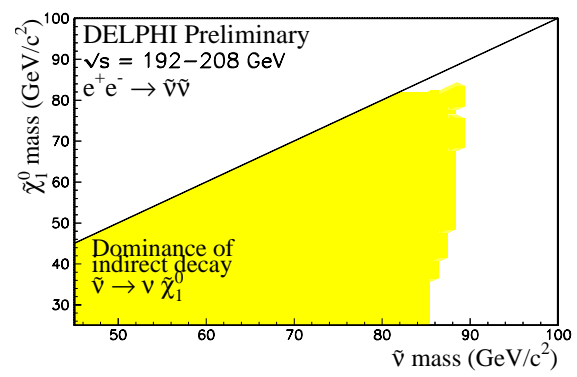

Figure 3: Excluded region in the sneutrino-LSP mass plane considering sneutrino indirect RpV decay through a $\lambda$ type coupling. 


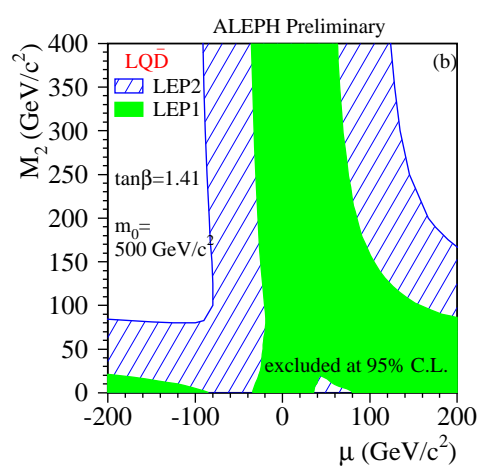

Figure 4: Excluded region in the $\left(\mathrm{M}_{2}, \mu\right)$ plane, obtained for $\tan \beta=1.4$ and a high value of $\mathrm{m}_{0}$, assuming the presence of a $\lambda^{\prime} \mathrm{RpV}$ coupling.
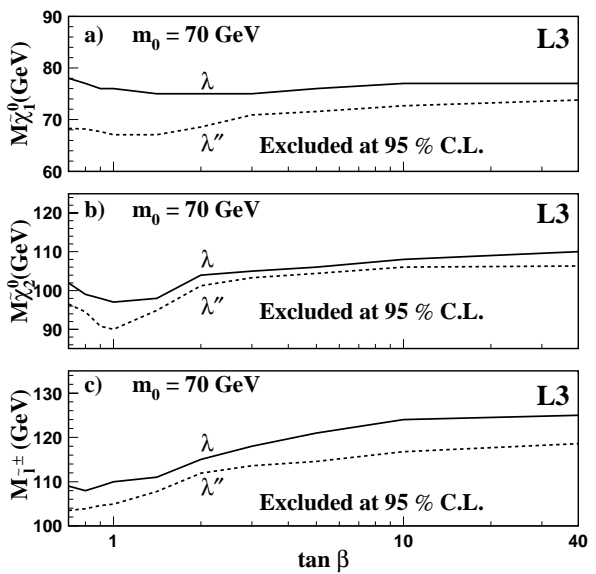

Figure 5: Limits on the sparticle masses as a function of $\tan \beta$ considering a low value of $\mathrm{m}_{0}$ and $\mathrm{RpV}$ couplings of type $\lambda$ and $\lambda^{\prime \prime}$.

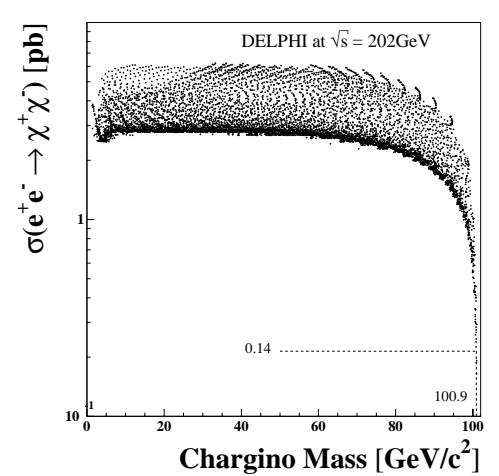

Figure 6: Expected chargino pair production cross-section at $202 \mathrm{GeV}$ (dots) as a function of the chargino mass (the dots correspond to scans in the MSSM parameters. A high $\mathrm{m}_{0}$ was assumed.) The obtained cross-section limit of $0.14 \mathrm{pb}^{-1}$ translates into a limit of $100.9 \mathrm{GeV} / \mathrm{c}^{2}$ in the chargino mass. 